\title{
Influence of the Distance between Nanoparticles in Clusters on the Magnetization Reversal Process
}

\author{
Andrea Ehrmann ${ }^{1}$ and Tomasz Blachowicz ${ }^{2}$ \\ ${ }^{1}$ Faculty of Engineering and Mathematics, Bielefeld University of Applied Sciences, Interaktion 1, 33619 Bielefeld, Germany \\ ${ }^{2}$ Institute of Physics-Center for Science and Education, Silesian University of Technology, Ul. Konarskiego 22B, 44-100 Gliwice, Poland
}

Correspondence should be addressed to Andrea Ehrmann; andrea.ehrmann@fh-bielefeld.de

Received 27 March 2017; Accepted 12 July 2017; Published 20 August 2017

Academic Editor: Anil Annadi

Copyright (C) 2017 Andrea Ehrmann and Tomasz Blachowicz. This is an open access article distributed under the Creative Commons Attribution License, which permits unrestricted use, distribution, and reproduction in any medium, provided the original work is properly cited.

Fourfold magnetic nanoparticles, created from nanowires or in the form of an open square, offer the possibility of creating quaternary memory devices with four unambiguously distinguishable stable states at remanence. This feature, however, has been simulated for single magnetic nanoparticles or clusters with interparticle distances similar to the nanoparticle dimensions. For the possible use in bit-patterned media, it is important to understand the scaling behavior of the stability of the additional intermediate states with the interparticle distance. The paper investigates exemplarily nanoparticles of two shapes which were found to be optimum to gain four states at remanence. For clusters of these particles, the probability of reaching the additional intermediate states in all particles in the same field region is strongly reduced with decreased interparticle distance. The differences between both shapes indicate possible solutions for this problem in the form of new nanoparticle shapes.

\section{Introduction}

Magnetic nanoparticles are of technological interest, for example, for magnetic storage media, magnetic sensors, and MRAMs [1-3]. Since their overall anisotropy is governed by the shape anisotropy [4], tailoring a nanoparticle's form allows for adjusting its magnetic properties. This is important for the possible use of nanoparticles in bit-patterned media or other applications in which defined anisotropies are necessary. One possible shape consists of a fourfold ring with different shape modifications, leading to two additional magnetic states at remanence which could be used to create quaternary memory systems [5]. In this way, data storage density could be increased by increasing the number of bits per particle instead of increasing the particle density. This approach has also been followed by other groups, resulting in three, four, or up to eight magnetization states in diverse nanostructures [6-10].

Problematic in all these magnetic systems, however, is the interaction between neighboring nanostructures, often strongly modifying the single nanoparticle's magnetic properties. For multilayer systems, for example, distances of $30 \mathrm{~nm}$ were found to be sufficient to approximate independent magnetic behavior of the layers [11]. In an array of nanotubes with distances of $30 \mathrm{~nm}$ or $60 \mathrm{~nm}$, however, even the larger distance showed a significant influence of the cluster structure on magnetization reversal, especially by nearly completely annihilating a step in the hysteresis loop which occurred for single nanotubes of identical geometry [12]. Since such a step is also necessary for reaching stable intermediate states at remanence, this finding indicates possible problems with downscaling the fourfold ring structure under investigation here. For a large cluster of nanodots, the geometric shape in which the lowest stray fields and thus the smallest influences between nanoparticles can be expected, nearly random magnetization orientations were found lately [13].

Nevertheless, for the systems under investigation here, it was found in an another project that nanoparticles with diameters of $120 \mathrm{~nm}$ and identical interparticle distances did nearly not change their coercive fields and retained a collective intermediate onion state which is necessary for the creation of quaternary memory devices [14]. Due to the necessity to further minimize such nanoparticle clusters, it is, however, necessary to investigate the influence of further 


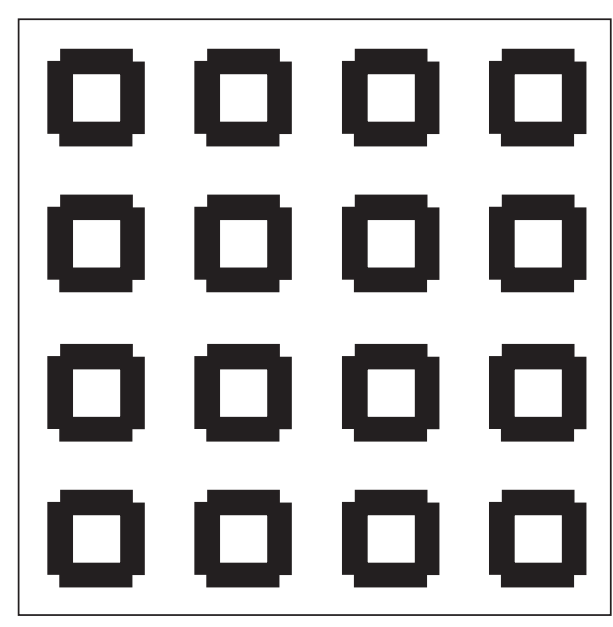

(a)

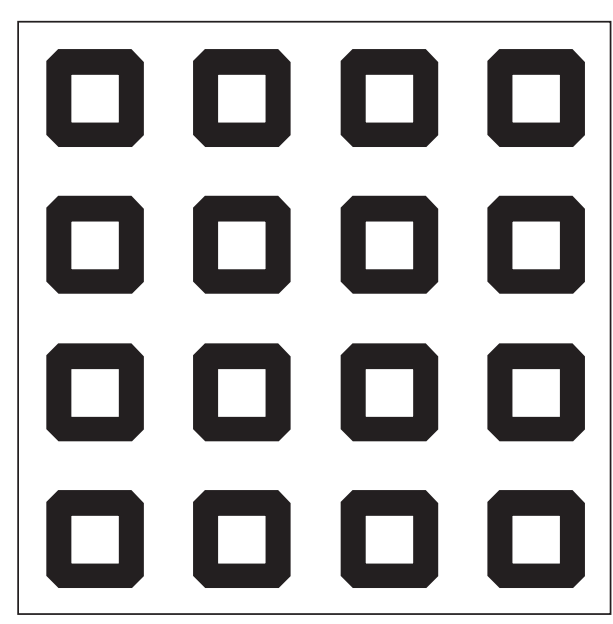

(b)

FIGURE 1: Clusters of $4 \times 4$ fourfold nanoparticles, showing two different shapes, here defined as "square corner cuts" (a) and "diagonal corner cuts" (b).

reduced distances between the single particles of specific shape, ordered in a cluster.

\section{Materials and Methods}

Modelling was performed using the micromagnetic simulator OOMMF (Object Oriented MicroMagnetic Framework) [15]. This program is based on finite differences (discretizing space into small cuboids) and utilizes the Landau-Lifshitz-Gilbert (LLG) equation of motion [16].

All simulations were performed using iron $(\mathrm{Fe})$ which has proven to show ideal properties in terms of stable intermediate states [17]. The following parameters were used for modelling, being consistent with typical literature values [18]: saturation magnetization $M_{S}=1700 \cdot 10^{3} \mathrm{~A} / \mathrm{m}$, exchange constant $A=21 \cdot 10^{-12} \mathrm{~J} / \mathrm{m}$, anisotropy constant $K_{1}=$ $48 \cdot 10^{3} \mathrm{~J} / \mathrm{m}^{3}$, Gilbert damping constant $\alpha=0.1$, and mesh diameter $d=5 \mathrm{~nm}$. Since such samples are mostly produced by sputtering in reality, the anisotropy was assumed to have randomly oriented axes in each cell.

While the external magnetic field was applied parallel to the sample plane, the orientation of the sample was varied between $0^{\circ}$ (defined as parallel orientation of the external magnetic field to the $x$-axis of the sample, i.e., to one side of the nanoparticle) and $45^{\circ}$ in steps of $5^{\circ}$. To ensure that magnetization saturation is reached, external fields were swept within $\pm 0.25 \mathrm{~T}$.

For the simulation, clusters of $4 \times 4$ particles were modelled. The particles under simulation here had slightly different geometries (Figure 1): one of them, defined as "square corner cuts," has an ideal structure which has shown to lead to two additional intermediate states at remanence [5], while the other one, denoted as "diagonal corner cuts," has a more realistic shape, as recognized in former lithographically produced nanostructures $[19,20]$. Lateral dimensions of each nanoparticle were $120 \mathrm{~nm}$ in the sample plane and $5 \mathrm{~nm}$ height, corresponding to the smallest samples used in former experimental investigations $[19,20]$. This value was chosen since the aim of this study is to find the highest possible particle density and thus the highest data density. Distances between the nanoparticles were reduced stepwise from $60 \mathrm{~nm}$ ( $1 / 2$ of the nanoparticle diameters) to $12 \mathrm{~nm}(1 / 10$ of the nanoparticle diameters).

For simulations of single particles, the shapes used here result, amongst others, in the magnetic states depicted in Figure 2. From positive saturation, magnetization switches in a first step into a horseshoe state and afterwards into an onion state. This onion state can be relaxed to zero external magnetic field, resulting in an intermediate, stable state at remanence. Proceeding further to negative saturation, a second horseshoe state becomes visible before finally negative saturation is reached.

Depending on the angle and the exact shape of the single nanoparticle, the horseshoe states may vanish. Intermediate onion states, correlated with stable additional states at remanence, occur in both systems usually in an angular region between $1^{\circ}$ and $15^{\circ}$. Additionally, the onion states usually occur in a wider field range than the horseshoe states and are thus of higher technological interest than the horseshoe states and therefore investigated here.

\section{Results and Discussion}

In Figure 3, hysteresis loops are visible, which are simulated for a sample orientation of $5^{\circ}$ on nanoparticles with diagonal corner cuts. While the hysteresis loops for the single particle (black line) and for the $4 \times 4$ cluster with interparticle distances half of the particle diameters (red line) are nearly identical, the cluster with interparticle distances of only a quarter of the particle diameters (green line) results in a significantly modified hysteresis loop. Apparently the interaction between particles spaced by distances of half the particle size can still be neglected, while significant interactions occur at smaller distances. 


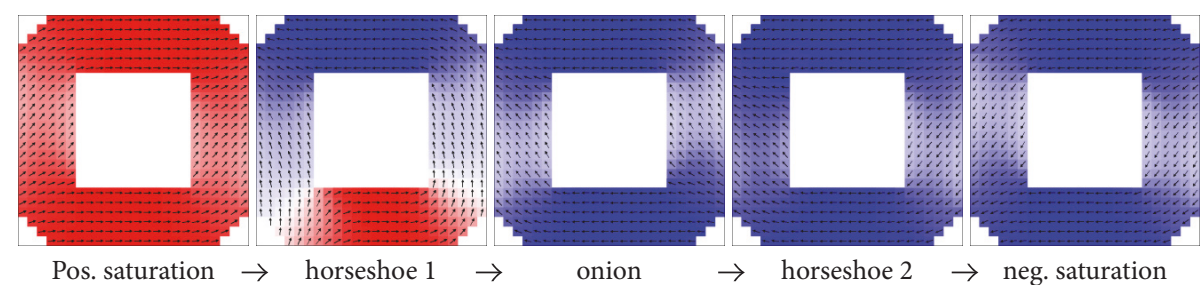

FIgURE 2: Typical magnetization reversal process for single particles at small angles (with or without horseshoe states possible, here shown for $5^{\circ}$, particle size $120 \mathrm{~nm}$ ).

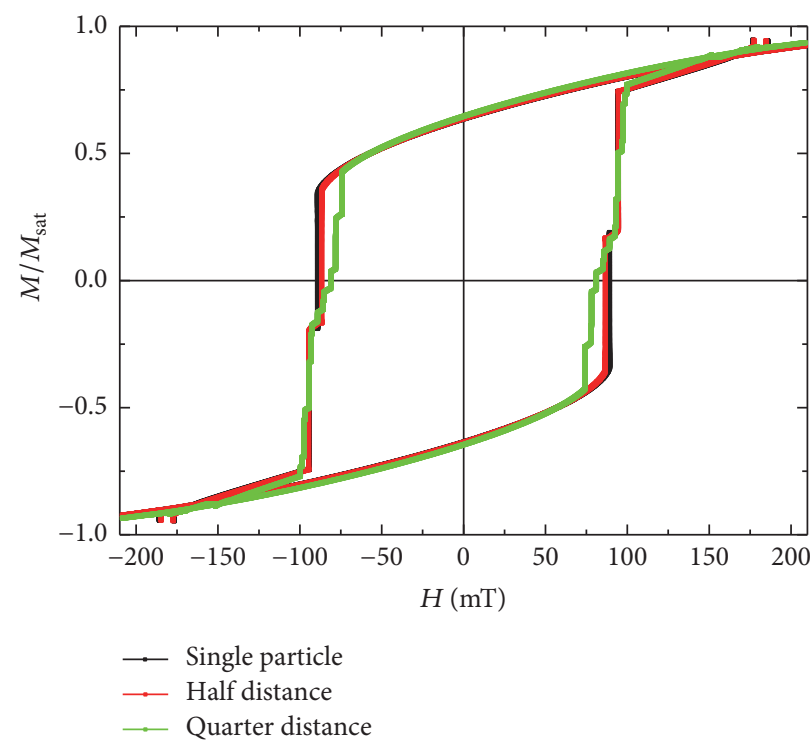

FIGURE 3: Hysteresis loops, simulated for nanoparticles with diagonal corner cuts at $5^{\circ}$, here depicted for a single particle (black line) and clusters with interparticle distances half of the particle diameters (red line) or a quarter of the particle diameters (green line).

Due to this finding, the simulated clusters were modified with respect to their interparticle distances in a range between half the particle dimension and $1 / 8$ of the particle dimension. In Figure 4, the results for the nanoparticles with diagonal corner cuts can be found.

Firstly, the coercive fields are only slightly modified between the four different situations. The magnetic states, however, change significantly.

For the single particle, at small angles the magnetization reversal proceeds as depicted in Figure 2: Directly at the coercive field, a first horseshoe state is reached, followed by an onion state and a second horseshoe state, marked as blue bar, green bar, and dark blue bar, respectively, in Figure 4. At $20^{\circ}$, the second horseshoe state vanishes; at higher angles, both other states vanish, too, and magnetization reversal occurs via a $180^{\circ}$ flip from positive to negative saturation without stable intermediate states. The "blank" areas are correlated with mixed states in which some of the particles of the cluster show one magnetization state and other particles are in another state.

For the cluster with interparticle distances of half the nanoparticle dimensions, the second horseshoe state is completely gone, and the onion state as well as the first horseshoe state is significantly reduced. It should be mentioned that, in all graphs, only the areas in which all particles in the cluster show the identical state are marked as belonging to the respective magnetization states.

Proceeding to $1 / 4$ interparticle distance (i.e., $30 \mathrm{~nm}$ ), the field regions in which all cluster particles are simultaneously in the first horseshoe or the onion state are further reduced. At the 1/8 interparticle distance, finally, only for one angle the onion state is retained, although with a significantly reduced field region.

Similar results can be found in the simulations of particles with square corner cuts, as depicted in Figure 5. Here, additional states occur, building a vortex with one or two domain walls. While the original single nanoparticle offers a broad spectrum of different magnetic states, most of the coherent states in the higher angle regime are already vanished for the cluster with distances identical to half the particle diameter. Decreasing the interparticle distances further, the coherent states become smaller again, until they completely vanish for the cluster with interparticle distances of $1 / 8$ of the particle diameters.

Figure 6 shows the widths of the field areas in which all nanoparticles of a cluster are in the onion state at a field orientation of $5^{\circ}$, depicted as a function of the inverse interparticle distance. For both nanoparticle shapes, the 

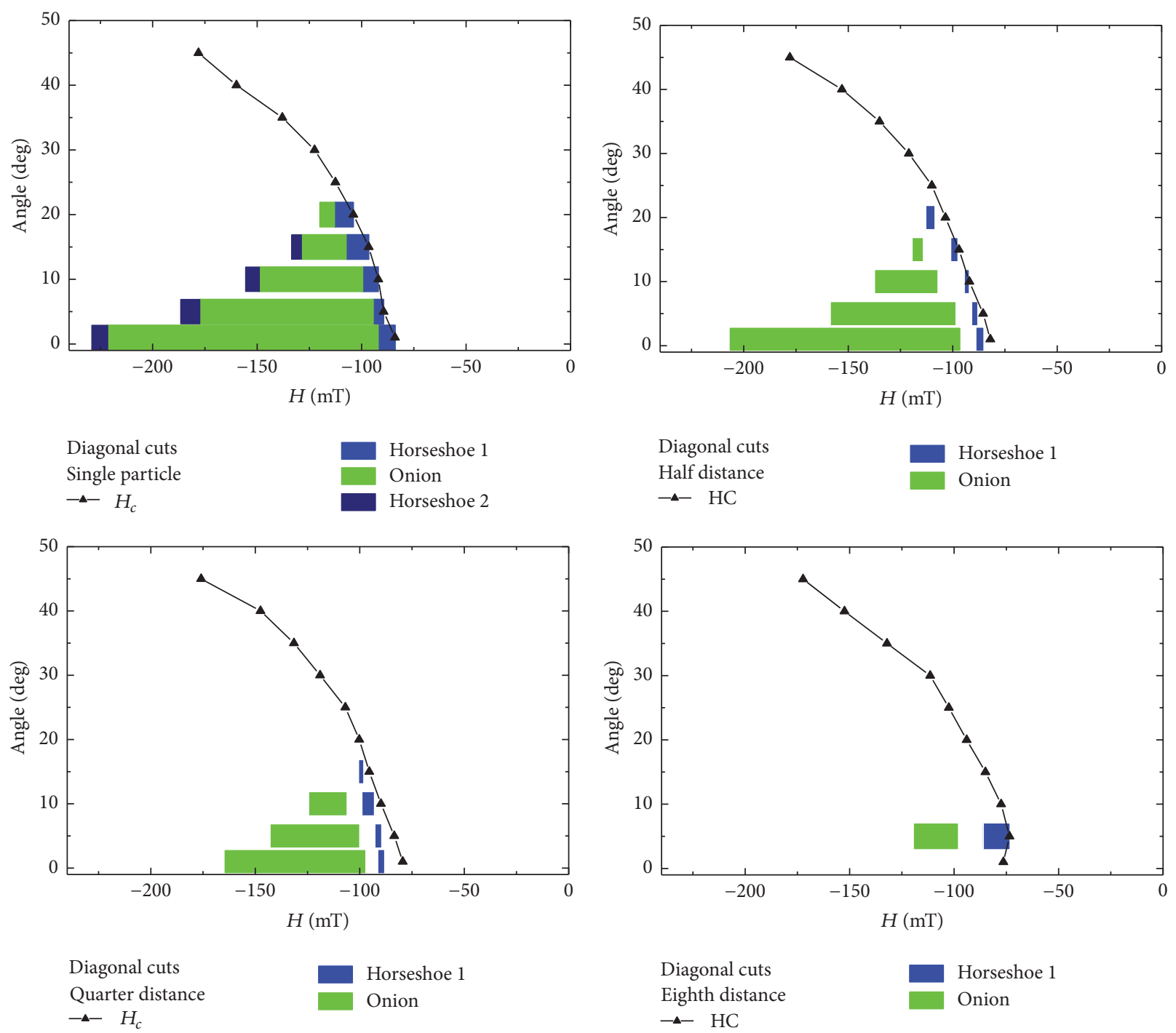

Figure 4: Coercive fields and field regions of different coherent magnetic states, as described in the text, for samples with diagonal corner cuts.

correlation is not exactly linear. As derived from Figures 4 and 5, the clusters with diagonal corner cuts show coherent behavior for smaller interparticle distances; however, for a distance of $12 \mathrm{~nm}$ between the particles $(=1 / 10$ of the nanoparticle diameter), the onion state vanishes here, too.

Thus, additional nanoparticle shapes were tested by building $4 \times 4$ clusters with $12 \mathrm{~nm}$ interparticle distances and simulating their magnetization reversal behavior for a sample orientation of $5^{\circ}$. This orientation was chosen in agreement with Figure 4 which showed that here the coherent behavior was kept the longest. Figure 7 depicts a few of the tested clusters with the corresponding widths of the coherently ordered onion states. As can be seen here, not only are inner and outer shapes of the nanoparticles of importance, but also their orientation towards the neighbors is crucial. This suggests that biomimetic structures, such as the Fibonacci spirals [21] well-known from sunflowers and several other plants and animals, may be an interesting approach to create nanoparticle patterns in which the interactions between neighboring particles are as small as possible.

\section{Conclusions}

Using micromagnetic simulations, we have shown that even for simulated, and thus quite symmetric, particles forming clusters, the dipolar-like interactions between them strongly disturb the formation of coherently ordered magnetization states in which all particles are in the same magnetic state. Particularly, the onion state often found in fourfold nanoparticles with open core, which is of technological interest due to the possibility of using such particles in quaternary memory devices, cannot be maintained for too small distances between neighboring particles.

By modification of shapes and arrangement of the original nanoparticle clusters, new structures could be found in which the particles of $120 \mathrm{~nm}$ could be approached to 

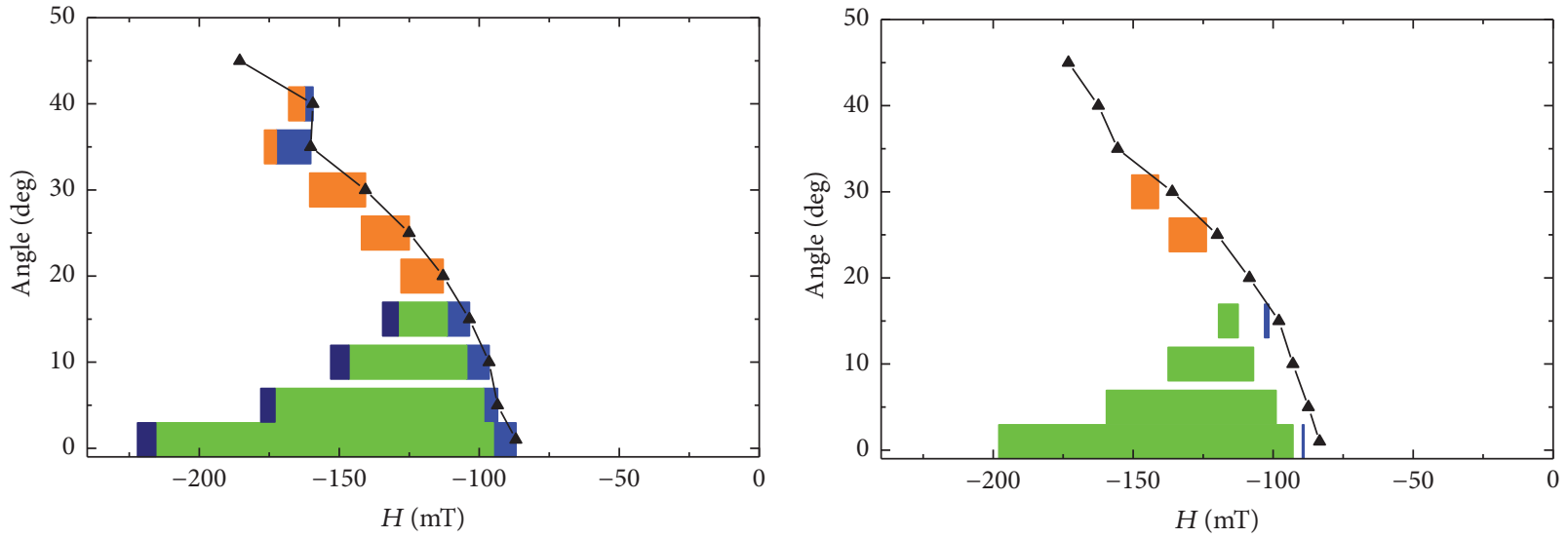
Square cuts
Single particle
$-\triangle-H_{c}$

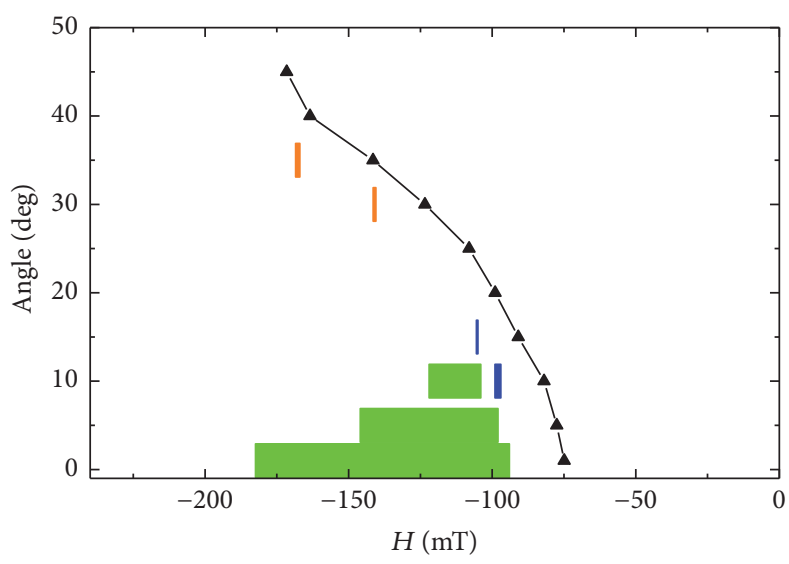

Square cuts Quarter distance $-\mathbf{-} H_{c}$
Horseshoe 1

Onion

DW vortex

Horseshoe 2

Horseshoe 1
Onion
DW vortex

Square cuts Horseshoe 1

Half distance Horseshoe 2

-^- HC DW vortex

Onion

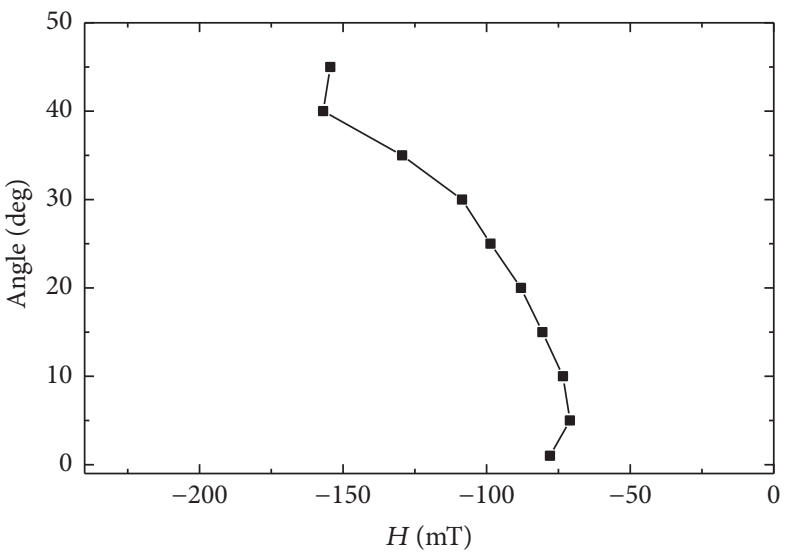

Square cuts

Eighth distance

$--H_{c}$

FIGURE 5: Coercive fields and field regions of different coherent magnetic states, as described in the text, for samples with square corner cuts.

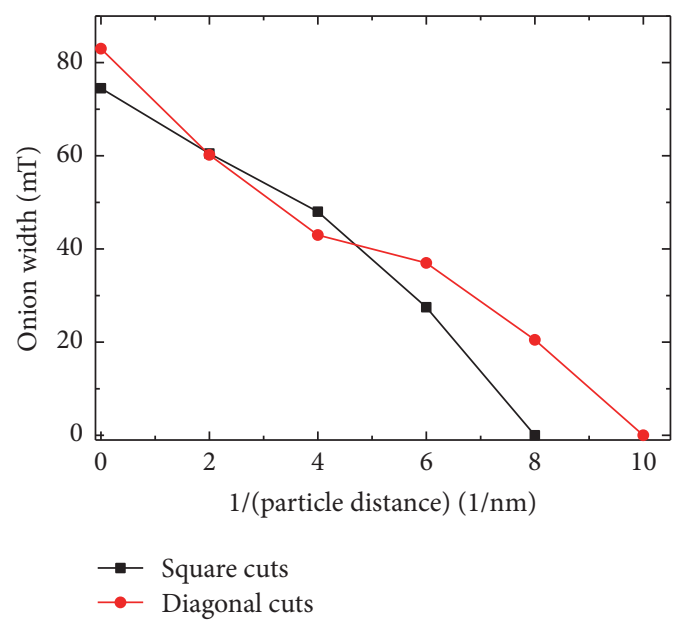

FIGURE 6: Field areas in which onion states occur coherently for clusters investigated at $5^{\circ}$ versus the inverse interparticle distances. 


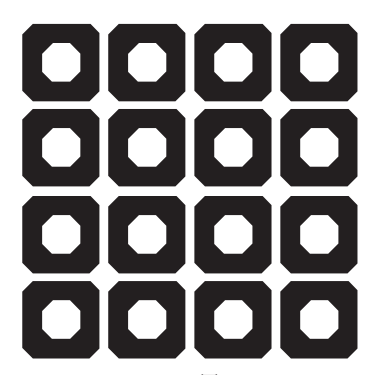

$1 \mathrm{mT}$

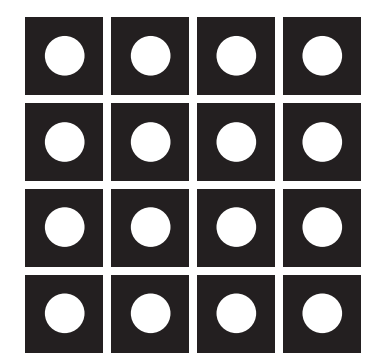

$6 \mathrm{mT}$

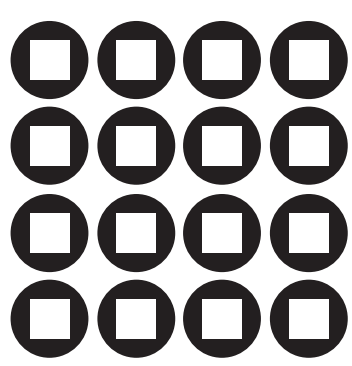

$0 \mathrm{mT}$

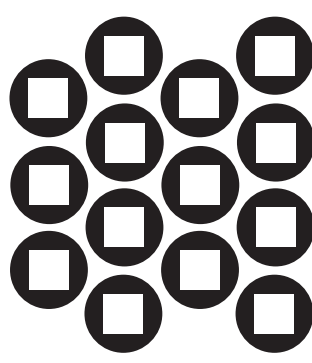

$7.5 \mathrm{mT}$

FIGURE 7: Different clusters under simulation, consisting in most cases of $4 \times 4$ particles with interparticle distances of $12 \mathrm{~nm}$, and respective widths of coherent onion states.

distances of $12 \mathrm{~nm}$ without losing the coherent onion state. These findings suggest new solutions to design clusters of independent magnetic nanoparticles.

\section{Conflicts of Interest}

The authors declare that there are no conflicts of interest regarding the publication of this paper.

\section{References}

[1] C. Vogler, F. Bruckner, M. Fuger et al., "Three-dimensional magneto-resistive random access memory devices based on resonant spin-polarized alternating currents," Journal of Applied Physics, vol. 109, no. 12, article 123901, 2011.

[2] U. Roy, H. Seinige, F. Ferdousi, J. Mantey, M. Tsoi, and S. K. Banerjee, "Spin-transfer-torque switching in spin valve structures with perpendicular, canted, and in-plane magnetic anisotropies," Journal of Applied Physics, vol. 111, no. 7, article 07C913, 2012.

[3] S. Yoon, Y. Jang, Ch. Nam et al., "Sensitivity enhancement of a giant magnetoresistance alternating spin-valve sensor for highfield applications," Journal of Applied Physics, vol. 111, article 07E504, 2012.

[4] J. Åkerman, “Toward a universal memory," Science, vol. 308, no. 5721, pp. 508-510, 2005.

[5] T. Blachowicz and A. Ehrmann, "Fourfold nanosystems for quaternary storage devices," Journal of Applied Physics, vol. 110, no. 7, article 073911, 2011.

[6] W. Zhang and S. Haas, "Phase diagram of magnetization reversal processes in nanorings," Physical Review B, vol. 81, article 064433, 2010.

[7] R.-H. Wang, J.-S. Jiang, and M. Hu, "Metallic cobalt microcrystals with flowerlike architectures: synthesis, growth mechanism and magnetic properties," Materials Research Bulletin, vol. 44, no. 7, p. 1468, 2009.

[8] L. Thevenard, H. T. Zeng, D. Petit, and R. P. Cowburn, "Macrospin limit and configurational anisotropy in nanoscale permalloy triangles," Journal of Magnetism and Magnetic Materials, vol. 322, no. 15, pp. 2152-2156, 2010.

[9] L. Huang, M. A. Schofield, and Y. Zhu, "Control of doublevortex domain configurations in a shape-engineered trilayer nanomagnet system," Advanced Materials, vol. 22, article 492, 2010 .
[10] J. Moritz, G. Vinai, S. Auffret, and B. Dieny, "Two-bit-perdot patterned media combining in-plane and perpendicular-toplane magnetized thin films," Journal of Applied Physics, vol. 109, article 083902, 2011.

[11] R. A. Escobar, S. Castillo-Sepulveda, S. Allende et al., "Towards independent behavior of magnetic slabs," IEEE Magnetics Letters, vol. 8, pp. 1-5, 2017.

[12] D. Salazar-Aravena, J. L. Palma, and J. Escrig, "Magnetostatic interactions between wire-tube nanostructures," Journal of Applied Physics, vol. 117, no. 19, article 193905, 2015.

[13] B. Van De Wiele, S. Fin, A. Sarella, P. Vavassori, and D. Bisero, "How finite sample dimensions affect the reversal process of magnetic dot arrays," Applied Physics Letters, vol. 105, no. 16, article 162407, 2014.

[14] A. Ehrmann and T. Blachowicz, "Interaction between magnetic nanoparticles in clusters," AIMS Materials Science, vol. 4, pp. 383-390, 2017.

[15] M. J. Donahue and D. G. Porter, "OOMMF Users Guide, Version 1.0," Interagency Report NISTIR 6376, National Institute of Standards and Technology, Gaithersburg, MD, USA, 1999.

[16] T. L. Gilbert, "A phenomenological theory of damping in ferromagnetic materials," IEEE Transactions on Magnetics, vol. 40, no. 6, pp. 3443-3449, 2004.

[17] A. Ehrmann and T. Blachowicz, "Influence of shape and dimension on magnetic anisotropies and magnetization reversal of $\mathrm{Py}, \mathrm{Fe}$, and Co nano-objects with four-fold symmetry," AIP Advances, vol. 5, no. 9, article 097109, 2015.

[18] E. F. Kneller and R. Hawig, "The exchange-spring magnet: a new material principle for permanent magnets," IEEE Transactions on Magnetics, vol. 27, no. 4, pp. 3588-3600, 1991.

[19] A. Ehrmann, T. Blachowicz, S. Komraus et al., "Magnetic properties of square Py nanowires: irradiation dose and geometry dependence," Journal of Applied Physics, vol. 117, no. 17, Article ID 173903, 2015.

[20] A. Ehrmann, S. Komraus, T. Blachowicz et al., "Pseudo exchange bias due to rotational anisotropy," Journal of Magnetism and Magnetic Materials, vol. 412, pp. 7-10, 2016.

[21] T. O. Omotehinwa and S. O. Ramon, "Fibonacci numbers and golden ratio in mathematics and science," International Journal of Computer and Information Technology, vol. 2, no. 4, article 630, 2013. 

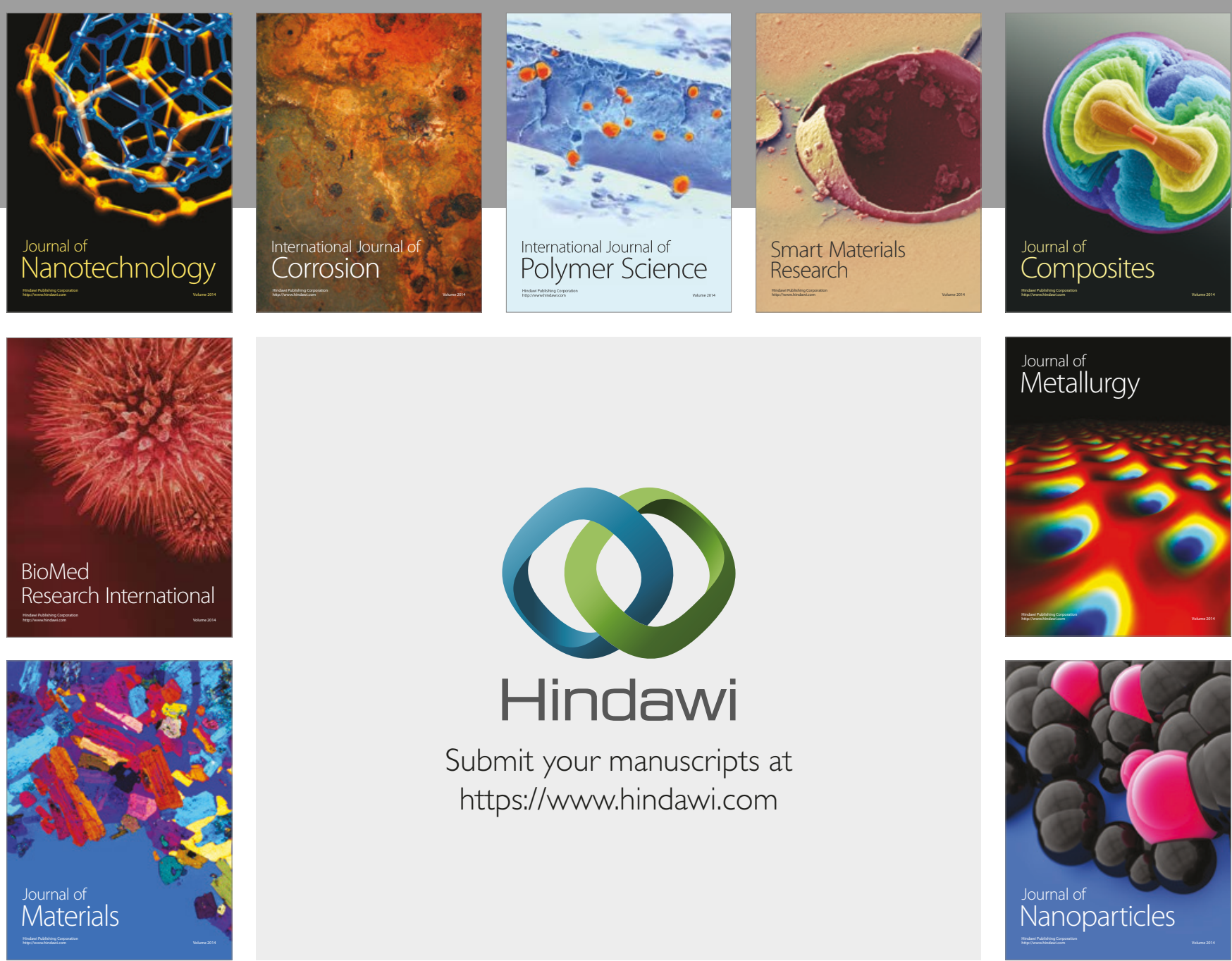

\section{Hindawi}

Submit your manuscripts at

https://www.hindawi.com
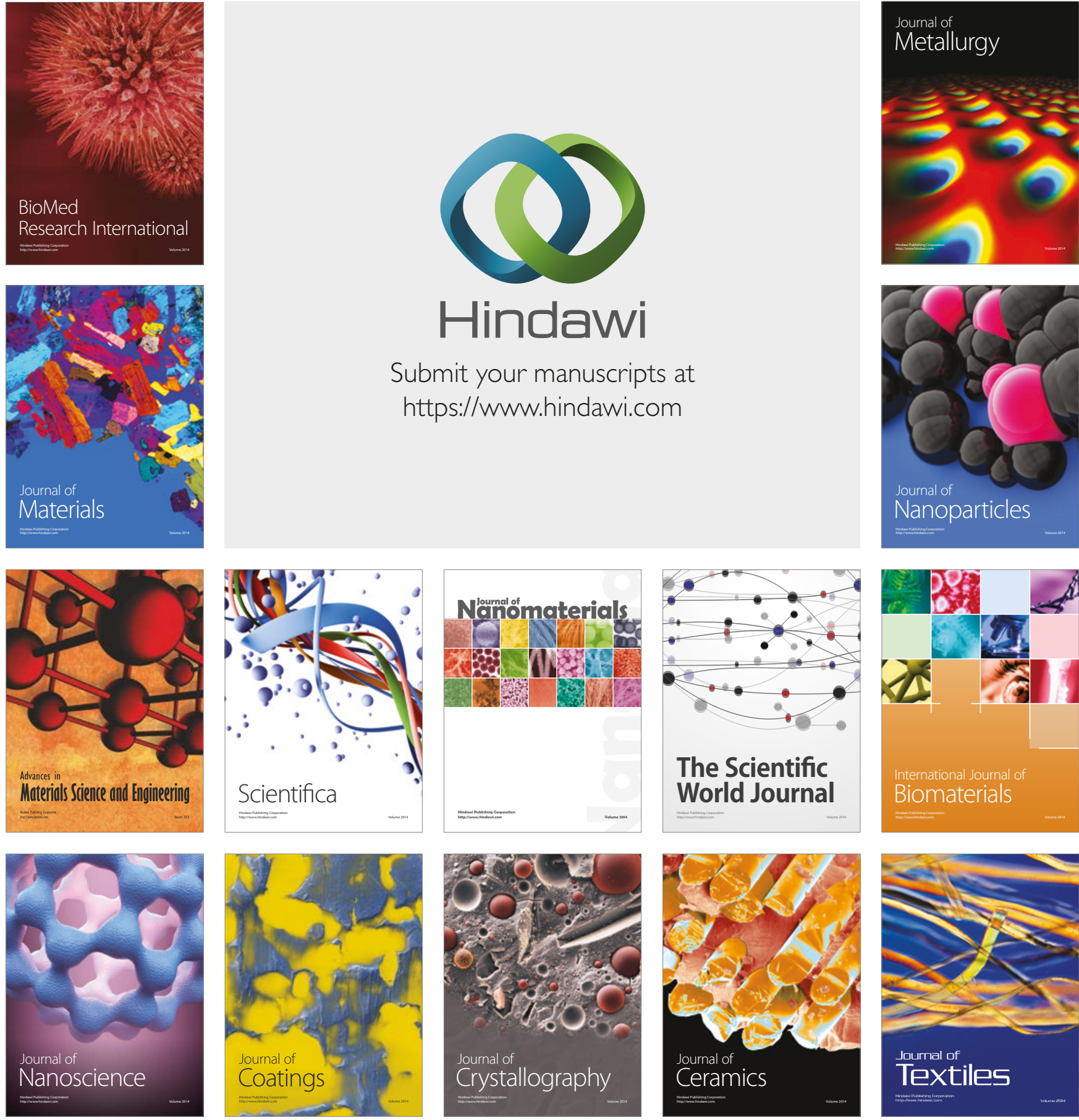

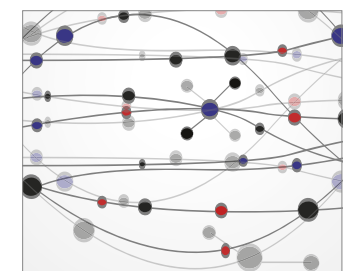

The Scientific World Journal
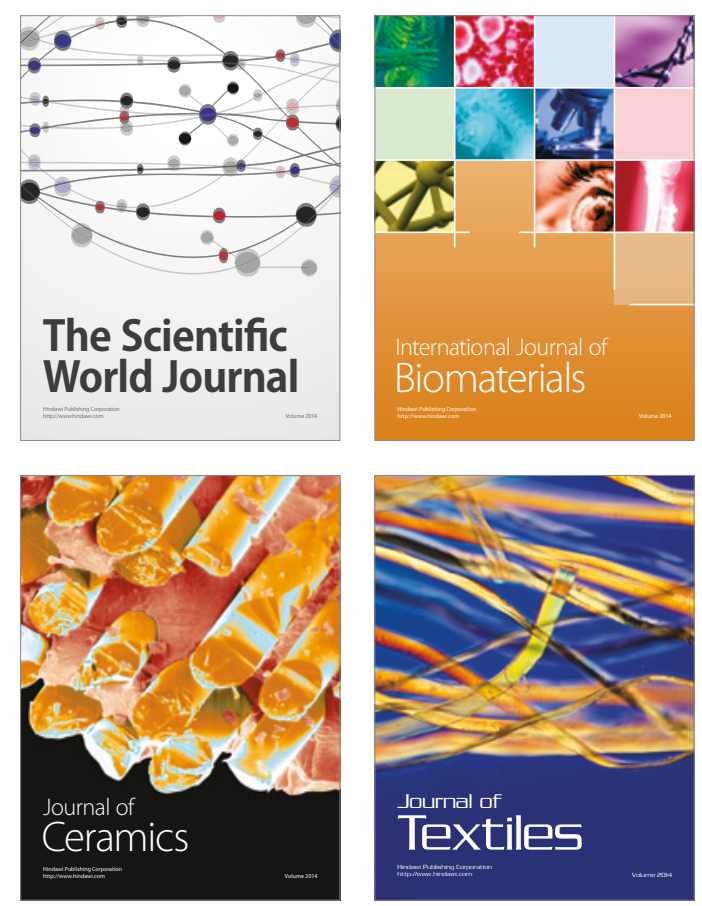